\title{
IMPROVING THE ACCEPTANCE RATE OF REVERSIBLE JUMP MCMC PROPOSALS
}

\author{
FAHIMAH Al-AwAdhi * MERrileE HurN ${ }^{\dagger}$ \\ CHRISTOPHER JENNISON $\ddagger$
}

May 1, 2002

\begin{abstract}
Recent articles have commented on the difficulty of proposing efficient reversible jump moves within MCMC. We suggest a new way to make proposals more acceptable using a secondary Markov chain to modify proposed moves - at little extra programming cost.
\end{abstract}

KEYWORDS: Image analysis; Markov chain Monte Carlo; Object recognition; Reversible jump MCMC; Stochastic simulation; Tempering.

\section{INTRODUCTION}

Since 1970, when Hastings introduced Markov chain Monte Carlo algorithms into the statistics literature, the use of Markov chain simulation has grown enormously and become an almost routine tool in some application areas (Hastings, 1970, Gilks et al, 1995). The appeal and applicability of these methods received another boost with the extension to distributions defined over variable dimensions, reversible jump MCMC (Green, 1995). Implementations of this RJMCMC methodology generally use a selection of move types, some of which explore the sample space within a fixed dimension while others make changes in dimensionality. Both types of move

\footnotetext{
${ }^{*}$ Department of Statistics and OR, Kuwait University, P.O.Box 5969 Safat, Kuwait, 13060

${ }^{\dagger}$ Corresponding author: Department of Mathematics, University of Bristol, Bristol, BR8 1TW

${ }^{\ddagger}$ Department of Mathematical Sciences, University of Bath, Claverton Down, Bath, BA2 7AY
} 
should be as efficient as possible in moving around the sample space. The first sign of inefficiency is an unacceptably high rejection rate for the proposed changes.

In fixed dimensions, small changes usually have higher acceptance rates than large changes and proposal mechanisms can be scaled to achieve a desired acceptance rate. Brooks, Giudici and Roberts (2001) point out that this option is not always available for moves between dimensions as there is often no natural distance measure between states of different dimensions, for example, these may correspond to competing nonnested models with different sets of parameters.

In this article we consider highly structured problems where jumps must be made between multi-modal distributions in different dimensional spaces. We focus on some of the severe problems that occur in sampling high-level posterior image distributions. We suggest an approach which makes use of a secondary Markov chain to modify an initial proposal before taking the acceptance decision. This allows the proposal to move from a low-probability region of the new space towards a mode before a comparison is made with the starting state. The approach has the benefit that existing code may often be used to run the secondary chain. In Section 2, we describe the reversible jump framework, the problem of slow movement across different dimensions, and approaches suggested to alleviate this problem. In Section 3, we describe our new approach and the circumstances in which it is likely to be particularly beneficial. We present a simple example to illustrate the method's potential in Section 4 and an object recognition example in Section 5.

\section{The REVERSible Jump FrAMEWORK}

Suppose we wish to sample from a distribution $\pi(\boldsymbol{x})$ in which the vector $\boldsymbol{x}$ is of variable dimension. Let $\pi_{n}(\boldsymbol{x})$ denote the sub-density with respect to Lebesgue measure on $R^{n}$ for values of $\boldsymbol{x}$ with dimension $n$. Suppose $q_{m n}\left(\boldsymbol{x}, \boldsymbol{x}^{\prime}\right)$ is a proposal density on $R^{n}$, or a subspace of $R^{n}$, for moves from $R^{m}$ to $R^{n}$ and the reverse proposal for moving from $R^{n}$ to $R^{m}$ has density $q_{n m}\left(\boldsymbol{x}^{\prime}, \boldsymbol{x}\right)$. (For $m<n$, transitions from $\boldsymbol{x} \in R^{m}$ to $\boldsymbol{x}^{\prime} \in R^{n}$ are often defined such that $\boldsymbol{x}$ determines $m$ elements of $\boldsymbol{x}^{\prime}$ and $q_{m n}\left(\boldsymbol{x}, \boldsymbol{x}^{\prime}\right)$ is a density on the remaining $n-m$ dimensional subspace of $R^{n}$; in this 
case, the reverse step is deterministic and the kernel $q_{n m}\left(\boldsymbol{x},{ }^{\prime} \boldsymbol{x}\right)$ degenerate.) Green (1995) gives the formula

$$
\alpha_{m n}\left(\boldsymbol{x}, \boldsymbol{x}^{\prime}\right)=\min \left\{1, \frac{\pi_{n}\left(\boldsymbol{x}^{\prime}\right) q_{n m}\left(\boldsymbol{x}^{\prime}, \boldsymbol{x}\right)}{\pi_{m}(\boldsymbol{x}) q_{m n}\left(\boldsymbol{x}, \boldsymbol{x}^{\prime}\right)}\right\}
$$

for the acceptance probability needed to maintain detailed balance with respect to $\pi$.

In any MCMC sampler, the acceptance rate of proposals should be reasonably high. In fixed dimension problems, a proposal of the form $\boldsymbol{x}^{\prime}=\boldsymbol{x}+\boldsymbol{\eta}$, where $\boldsymbol{\eta}$ is a zero-mean random variable, can be calibrated by adjusting the variance of $\boldsymbol{\eta}$ to achieve a suitable acceptance rate. Unfortunately, there is often no simple parallel to this approach when $\boldsymbol{x}$ and $\boldsymbol{x}^{\prime}$ have different dimensions.

Suggestions have been made for improving acceptance rates in RJMCMC. Green and Mira (2001) introduce a second stage to the proposal mechanism in their "delayed rejection sampler". Following rejection of a proposed move, a second proposal is made bearing in mind the first rejection and possibly making use of the rejected proposal value; the overall acceptance probability takes into account this more complicated structure. The method offers the option of proposing a large change in dimension and switching to a smaller change in dimension if this is rejected.

Brooks, Giudici and Roberts (2001) return to the scaling idea by looking for canonical jump functions which play the role of $\boldsymbol{\eta}=\mathbf{0}$ in terms of tuning the acceptance rate, but now acting across dimensions. For example, with nested models such as AR processes of unknown order, a canonical jump would be to increase the order by one, with the newly introduced coefficient equal to zero and all other coefficients unchanged. Once suitable correspondences have been identified, proposals can be constructed as scaled perturbations around these central moves. A second idea suggested by Brooks et al (2001) is that of using auxiliary variables to "pad-out" the problem to be of the same dimension for all models under consideration. These auxiliary variables retain information about a model even when that model is not the current one. This means that when a move back to that model is proposed, the auxiliary variables may be able to guide the proposal to somewhere close to where it last left.

In another approach, Green (2002) tries to regain some of the simplicity of the random walk Metropolis sampler with a view to automating implementation of RJMCMC methods. A trial run is used to approximate the mean and variance of $\boldsymbol{x}$ 
in each dimension. These values are then used in constructing appropriate dimension Gaussian proposals both within and between dimensions.

Stephens (2000) suggests an alternative to RJMCMC sampling of mixture distributions based on spatial birth and death processes. Here, the mixture components correspond to individual marked points in the point process and dimensionality changes are made using birth and death processes to simulate the point process. Although this approach can be generalised to other applications, it does not necessarily offer a solution to mixing problems: Cappe, Robert and Ryden (2001) note the close relationship between RJMCMC and spatial birth and death processes, and warn that problems affecting the former are liable to arise in the latter.

\section{Alternative Proposal mechanism}

When a target distribution is highly modal within each dimension, proposals which land away from the modal region have virtually no chance of acceptance. If the locations of modes are not initially known, designing a matched pair of dimensionchanging proposals to produce jumps from mode to mode requires much ingenuity, if indeed it is possible at all. Our approach is to modify the value of a simpler RJMCMC proposal, moving it closer to a mode before the accept-reject decision is taken.

Suppose the Markov chain sampler is currently in state $\boldsymbol{x} \in R^{m}$ and we have chosen to consider a jump to $R^{n}$. We first generate a proposal $\boldsymbol{x}^{\prime}$ in $R^{n}$ from the density $q_{m n}\left(\boldsymbol{x}, \boldsymbol{x}^{\prime}\right)$, just as before. But we now follow this by $k$ fixed-dimension MCMC steps through states $\boldsymbol{x}^{1}, \ldots, \boldsymbol{x}^{k}=\boldsymbol{x}^{*}$, each step satisfying detailed balance with respect to a new distribution $\pi_{n}^{*}(\boldsymbol{x})$. The final state $\boldsymbol{x}^{*} \in R^{n}$ is either accepted as the next state of the Markov chain or rejected, in which case the Markov chain remains at $\boldsymbol{x}$. In the reverse form of this move, a jump from $\boldsymbol{x}^{*} \in R^{n}$ to $R^{m}$ is proposed by taking $k$ fixed dimension steps within $R^{n}$, each of which satisfies detailed balance with respect to $\pi_{n}^{*}(\boldsymbol{x})$, ending at $\boldsymbol{x}^{\prime}$ say, then sampling from the density $q_{n m}\left(\boldsymbol{x}^{\prime}, \boldsymbol{x}\right)$ to give the proposal $\boldsymbol{x}$ for possible acceptance.

The acceptance probability must be calculated to ensure detailed balance with respect to the overall target distribution $\pi$. Let $P$ denote the transition kernel for the full 
sequence of $k$ moves in $R^{n}$ from $\boldsymbol{x}^{\prime}$ to $\boldsymbol{x}^{*}$. Assuming equal probabilities of choosing the two types of move from $R^{m}$ to $R^{n}$ and from $R^{n}$ to $R^{m}$, we require

$$
\begin{gathered}
\int_{\left(\boldsymbol{x}, \boldsymbol{x}^{\prime}, \boldsymbol{x}^{*}\right) \in A \times B \times C} \pi_{m}(\boldsymbol{x}) q_{m n}\left(\boldsymbol{x}, \boldsymbol{x}^{\prime}\right) P\left(\boldsymbol{x}^{\prime}, \boldsymbol{x}^{*}\right) \alpha_{m n}\left(\boldsymbol{x}, \boldsymbol{x}^{*}\right) d \boldsymbol{x} d \boldsymbol{x}^{\prime} d \boldsymbol{x}^{*}= \\
\int_{\left(\boldsymbol{x}, \boldsymbol{x}^{\prime}, \boldsymbol{x}^{*}\right) \in A \times B \times C} \pi_{n}\left(\boldsymbol{x}^{*}\right) P\left(\boldsymbol{x}^{*}, \boldsymbol{x}^{\prime}\right) q_{n m}\left(\boldsymbol{x}^{\prime}, \boldsymbol{x}\right) \alpha_{n m}\left(\boldsymbol{x}^{*}, \boldsymbol{x}\right) d \boldsymbol{x} d \boldsymbol{x}^{\prime} d \boldsymbol{x}^{*},
\end{gathered}
$$

for all Borel sets $A \in R^{m}, B \in R^{n}$ and $C \in R^{n}$ (or suitably defined sets $B(\boldsymbol{x})$ when $q_{m n}$ and $q_{n m}$ are defined on subspaces of $R^{n}$ and $\left.R^{m}\right)$. Then, the probability under $\pi$ of being in state $\boldsymbol{x}$, moving to $\boldsymbol{x}^{\prime}$ and on to $\boldsymbol{x}^{*}$ and accepting this proposal exactly balances the probability under $\pi$ of being in $\boldsymbol{x}^{*}$, making the reverse transitions to $\boldsymbol{x}^{\prime}$ and on to $\boldsymbol{x}$ and accepting this proposal.

One way to make the integrals equal is to make the integrands in (2) equal by taking

$$
\alpha_{m n}\left(\boldsymbol{x}, \boldsymbol{x}^{*}\right)=\min \left\{1, \frac{\pi_{n}\left(\boldsymbol{x}^{*}\right) P\left(\boldsymbol{x}^{*}, \boldsymbol{x}^{\prime}\right) q_{n m}\left(\boldsymbol{x}^{\prime}, \boldsymbol{x}\right)}{\pi_{m}(\boldsymbol{x}) q_{m n}\left(\boldsymbol{x}, \boldsymbol{x}^{\prime}\right) P\left(\boldsymbol{x}^{\prime}, \boldsymbol{x}^{*}\right)}\right\}
$$

and

$$
\alpha_{n m}\left(\boldsymbol{x}^{*}, \boldsymbol{x}\right)=\min \left\{1, \frac{\pi_{m}(\boldsymbol{x}) q_{m n}\left(\boldsymbol{x}, \boldsymbol{x}^{\prime}\right) P\left(\boldsymbol{x}^{\prime}, \boldsymbol{x}^{*}\right)}{\pi_{n}\left(\boldsymbol{x}^{*}\right) P\left(\boldsymbol{x}^{*}, \boldsymbol{x}^{\prime}\right) q_{n m}\left(\boldsymbol{x}^{\prime}, \boldsymbol{x}\right)}\right\} .
$$

Since each of the $k$ individual transitions which comprise $P$ satisfies detailed balance with respect to $\pi_{n}^{*}$, so does $P$. Hence, $\pi_{n}^{*}\left(\boldsymbol{x}^{*}\right) P\left(\boldsymbol{x}^{*}, \boldsymbol{x}^{\prime}\right)=\pi_{n}^{*}\left(\boldsymbol{x}^{\prime}\right) P\left(\boldsymbol{x}^{\prime}, \boldsymbol{x}^{*}\right)$ and (3) simplifies to

$$
\alpha_{m n}\left(\boldsymbol{x}, \boldsymbol{x}^{*}\right)=\min \left\{1, \frac{\pi_{n}\left(\boldsymbol{x}^{*}\right) \pi_{n}^{*}\left(\boldsymbol{x}^{\prime}\right) q_{n m}\left(\boldsymbol{x}^{\prime}, \boldsymbol{x}\right)}{\pi_{m}(\boldsymbol{x}) \pi_{n}^{*}\left(\boldsymbol{x}^{*}\right) q_{m n}\left(\boldsymbol{x}, \boldsymbol{x}^{\prime}\right)}\right\}
$$

There is an asymmetry in transitions in the two directions: in moves from $R^{m}$ to $R^{n}$ the jump across dimensions occurs first, then the MCMC steps under $\pi_{n}^{*}$; moves in the other direction start by taking steps within $R^{n}$ under $\pi_{n}^{*}$ and then jump to the proposal in $R^{m}$. With $m<n$, this may be appropriate if there is only a need to improve initial proposals in the higher dimensional space. If it is also desirable to modify the value reached on jumping to $R^{m}$ a spell of MCMC sampling under suitably modified target distributions could be included in both $R^{m}$ and $R^{n}$, either side of the reversible jump step, with a consequent change in the acceptance probability formula. 
It is instructive to compare likely values of $\alpha_{m n}\left(\boldsymbol{x}, \boldsymbol{x}^{\prime}\right)$ given by (1) for the original method and of $\alpha_{m n}\left(\boldsymbol{x}, \boldsymbol{x}^{*}\right)$ in (4) for the new method. Since the current state $\boldsymbol{x}$ has arisen in a Markov chain with equilibrium distribution $\pi$, we can expect $\boldsymbol{x}$ to take a typical value for the sub-distribution $\pi_{m}$. Assuming it is relatively easy to define a suitable transition to a lower dimension, we suppose $q_{n m}\left(\boldsymbol{x}^{\prime}, \boldsymbol{x}\right)$ will be comparable with $\pi_{m}(\boldsymbol{x})$. Difficulty arises in generating the additional elements in $\boldsymbol{x}^{\prime}$ in such a way that $q_{m n}\left(\boldsymbol{x}, \boldsymbol{x}^{\prime}\right)$ places weight on important parts of $\pi_{n}\left(\boldsymbol{x}^{\prime}\right)$ : if this is not achieved and $\boldsymbol{x}^{\prime}$ is a typical sample from $q_{m n}\left(\boldsymbol{x}, \boldsymbol{x}^{\prime}\right)$ but not from $\pi_{n}$, we have $\pi_{n}\left(\boldsymbol{x}^{\prime}\right) \ll q_{m n}\left(\boldsymbol{x}, \boldsymbol{x}^{\prime}\right)$ and the acceptance probability $\alpha_{m n}\left(\boldsymbol{x}, \boldsymbol{x}^{\prime}\right)$ in (1) will be extremely small. The difference in our new method is that the ratio $\frac{\pi_{n}\left(\boldsymbol{x}^{\prime}\right)}{q_{m n}\left(\boldsymbol{x}, \boldsymbol{x}^{\prime}\right)}$ in the old acceptance probability (1) is replaced by

$$
\frac{\pi_{n}\left(\boldsymbol{x}^{*}\right)}{\pi_{n}^{*}\left(\boldsymbol{x}^{*}\right)} \times \frac{\pi_{n}^{*}\left(\boldsymbol{x}^{\prime}\right)}{q_{m n}\left(\boldsymbol{x}, \boldsymbol{x}^{\prime}\right)}
$$

in the new $\alpha_{m n}\left(\boldsymbol{x}, \boldsymbol{x}^{*}\right)$ given by (4). The two factors in (5) are still liable to be less than 1 but a good choice of $\pi_{n}^{*}$, intermediate in some sense between $q_{m n}$ and $\pi_{n}$, can ensure that (4) is substantially larger than (1).

\section{A SIMPLE ILLUSTRATIVE EXAMPLE}

The following example demonstrates the scale of improvement our proposed method offers. Suppose $\pi$ is defined on $R^{1}$ and $R^{2}$ with $\pi_{1}$ equal to 0.5 times a $N(0,1)$ density on $R^{1}$ and $\pi_{2}$ equal to 0.5 times a bivariate normal density on $R^{2}$ in which $X_{1}$ and $X_{2}$ are independent with $X_{1} \sim N(0,1)$ and $X_{2} \sim N\left(0, \sigma^{2}\right)$. In proposing transitions from $\boldsymbol{x} \in R^{1}$ to $\boldsymbol{x}^{\prime} \in R^{2}$ we set $x_{1}^{\prime}=x_{1}$ and generate $x_{2}^{\prime}$ from a $N\left(0, \xi^{2}\right)$ distribution where $\xi^{2} \gg \sigma^{2}$; thus, this is an example of a proposal kernel $q_{12}$ which is poorly matched to $\pi_{2}$. For transitions from $\boldsymbol{x}^{\prime} \in R^{2}$ to $\boldsymbol{x} \in R^{1}$ we simply take $x_{1}=x_{1}^{\prime}$.

Consider first the standard reversible jump method when the typical value $x_{2}^{\prime}=\xi$ is proposed. (A more thorough treatment would integrate over the $N\left(0, \xi^{2}\right)$ distribution of $x_{2}^{\prime}$ but this simple case provides a simple, and compelling, illustration.) The factor in $\pi_{2}\left(\boldsymbol{x}^{\prime}\right)$ associated with $x_{1}^{\prime}$ is $\pi_{1}\left(x_{1}^{\prime}\right)$ and the remaining factor is a $N\left(0, \sigma^{2}\right)$ density evaluated at $x_{2}^{\prime}=\xi$. By definition, $q_{21}\left(\boldsymbol{x}^{\prime}, \boldsymbol{x}\right)=1$. In the denominator of (1) $\pi_{1}(\boldsymbol{x})=\pi_{1}\left(x_{1}^{\prime}\right)$ and $q_{12}\left(\boldsymbol{x}^{\prime}, \boldsymbol{x}\right)$ is a $N\left(0, \xi^{2}\right)$ density evaluated at $\xi$. Writing $f_{v}(w)$ to 
denote a $N(0, v)$ density evaluated at $w$, the acceptance probability of $\boldsymbol{x}^{\prime}$ is

$$
\begin{aligned}
\alpha_{12}\left(\boldsymbol{x}, \boldsymbol{x}^{\prime}\right)= & \min \left\{1, \frac{\pi_{2}\left(\boldsymbol{x}^{\prime}\right) q_{21}\left(\boldsymbol{x}^{\prime}, \boldsymbol{x}\right)}{\pi_{1}(\boldsymbol{x}) q_{12}\left(\boldsymbol{x}, \boldsymbol{x}^{\prime}\right)}\right\}=\frac{f_{1}\left(x_{1}\right) f_{\sigma^{2}}\left(x_{2}^{\prime}\right)}{f_{1}\left(x_{1}\right) f_{\xi^{2}}\left(x_{2}^{\prime}\right)} \\
& =\frac{f_{\sigma^{2}}(\xi)}{f_{\xi^{2}}(\xi)}=\frac{\xi}{\sigma} \exp \left\{-\frac{1}{2}\left(\frac{\xi^{2}}{\sigma^{2}}-1\right)\right\}
\end{aligned}
$$

and for $\xi^{2} \gg \sigma^{2}$ this probability can be very small.

Now consider our new method with $\pi_{2}^{*}$ defined as a bivariate normal density in which $X_{1}$ and $X_{2}$ are independent, $X_{1} \sim N(0,1)$ and $X_{2} \sim N\left(0, \phi^{2}\right)$ where $\sigma^{2}<\phi^{2}<\xi^{2}$. After an initial move to $\boldsymbol{x}^{\prime}$ where $x_{1}^{\prime}=x_{1}$ and $x_{2}^{\prime}$ takes the typical value $\xi$, the $k$ MCMC steps will lead to a new value $x_{1}^{*}$ of $x_{1}$ and a value $x_{2}^{*}$ sampled from $\pi_{2}^{*}$. Using the typical value $\phi$ for $x_{2}^{*}$, we obtain

$$
\begin{gathered}
\alpha_{12}\left(\boldsymbol{x}, \boldsymbol{x}^{*}\right)=\min \left\{1, \frac{\pi_{2}\left(\boldsymbol{x}^{*}\right) \pi_{2}^{*}\left(\boldsymbol{x}^{\prime}\right) q_{21}\left(\boldsymbol{x}^{\prime}, \boldsymbol{x}\right)}{\pi_{1}(\boldsymbol{x}) \pi_{2}^{*}\left(\boldsymbol{x}^{*}\right) q_{12}\left(\boldsymbol{x}, \boldsymbol{x}^{\prime}\right)}\right\}=\frac{f_{1}\left(x_{1}^{*}\right) f_{\sigma^{2}}\left(x_{2}^{*}\right) f_{1}\left(x_{1}\right) f_{\phi^{2}}\left(x_{2}^{\prime}\right)}{f_{1}\left(x_{1}\right) f_{1}\left(x_{1}^{*}\right) f_{\phi^{2}}\left(x_{2}^{*}\right) f_{\xi^{2}}\left(x_{2}^{\prime}\right)} \\
=\frac{f_{\sigma^{2}}(\phi) f_{\phi^{2}}(\xi)}{f_{\phi^{2}}(\phi) f_{\xi^{2}}(\xi)}=\frac{\xi}{\sigma} \exp \left\{-\frac{1}{2}\left(\frac{\xi^{2}}{\phi^{2}}+\frac{\phi^{2}}{\sigma^{2}}-2\right)\right\}
\end{gathered}
$$

Maximising (7) over $\phi^{2}$ gives the choice $\phi^{2}=\xi \sigma$ which we shall assume to be used. In this case

$$
\alpha_{12}\left(\boldsymbol{x}, \boldsymbol{x}^{*}\right)=\frac{\xi}{\sigma} \exp \left\{-\left(\frac{\xi}{\sigma}-1\right)\right\}
$$

Since only the square root of the ratio $\xi^{2} / \sigma^{2}$ now appears in the exponential term, this acceptance probability decreases much more slowly than (6) as $\xi^{2} / \sigma^{2}$ increases. A few numerical values of (6) and (8) are tabulated below.

$$
\begin{array}{cll}
\xi^{2} / \sigma^{2} & \alpha_{12}\left(\boldsymbol{x}, \boldsymbol{x}^{\prime}\right) & \alpha_{12}\left(\boldsymbol{x}, \boldsymbol{x}^{*}\right) \\
10 & 0.035 & 0.364 \\
25 & 0.00003 & 0.092 \\
50 & 1.6 \times 10^{-10} & 0.016 \\
100 & 3.2 \times 10^{-21} & 0.0012
\end{array}
$$

The clear conclusion is that our method can lead to a practically useful acceptance rate in situations where the original method is hopelessly slow. Further analysis of this example shows there can be additional gains from introducing a succession of target 
distributions intermediate between $q_{12}$ and $\pi_{2}$ : with $r-1$ suitably chosen intermediate distributions, the $r$ th root of $\xi^{2} / \sigma^{2}$ appears in the exponential in $\alpha_{12}\left(\boldsymbol{x}, \boldsymbol{x}^{\prime}\right)$.

In this example, $\pi^{*}$ is a "tempered" version of the target distribution $\pi$, at least as far as $X_{2}$ is concerned. Tempering is a natural way to create a flatter version of $\pi$ for the transition process to pass through and we use this in our object recognition example in the next section, tempering the conditional distribution of the components of interest. Sampling from a tempered version of $\pi$ can be based on the same fixeddimensional transitions used in sampling from $\pi$, hence little additional programming is needed beyond that required to implement the original reversible jump method. (For more discussion of tempering see Neal, 1996.)

\section{AN EXAMPLE IN OBJECT RECOGNITION}

We consider an object recognition example which first motivated our search for an improved proposal mechanism. The data $\boldsymbol{Y}$ displayed in Figure 1 were obtained by confocal microscopy. Our aim is to identify the number of the cells, $N$, in the scene and approximate their shapes by a set of ellipses $\boldsymbol{X}$. Using a Bayesian approach, inference is based on the posterior distribution of $N$ and $\boldsymbol{X}$ given the data $\boldsymbol{Y}=\boldsymbol{y}$. In line with our previous notation, we denote this posterior distribution $\pi(\boldsymbol{x})$ and let $\pi_{n}(\boldsymbol{x})$ denote the sub-density of the set of parameters for scenes with $n$ cells - now with respect to Lebesgue measure on $R^{6 n}$ since each cell has 6 parameters. Thus,

$$
\pi_{n}(\boldsymbol{x}) \propto \mathcal{L}(\boldsymbol{y} \mid n, \boldsymbol{x}) p_{n}(\boldsymbol{x})
$$

where $\mathcal{L}(\boldsymbol{y} \mid n, \boldsymbol{x})$ is the likelihood of the observed data given $N$ and $\boldsymbol{X}$, and $p_{n}$ is the density assigned to this scene in the prior model. Following the marked pointed process approach of Baddeley and van Lieshout (1993), we take the prior model to be a hard core interaction point process which prevents cells overlapping. The data $\boldsymbol{Y}$ are assumed to be Gaussian with different means and variances assigned to observations from different cells and from the background region; for more details see, for example, Rue and Hurn (1999) or Al-Awadhi (2001).

In MCMC sampling from $\pi$, several types of proposal are defined, including fixed dimensional moves which alter the size, shape or location of a single cell, as well as 


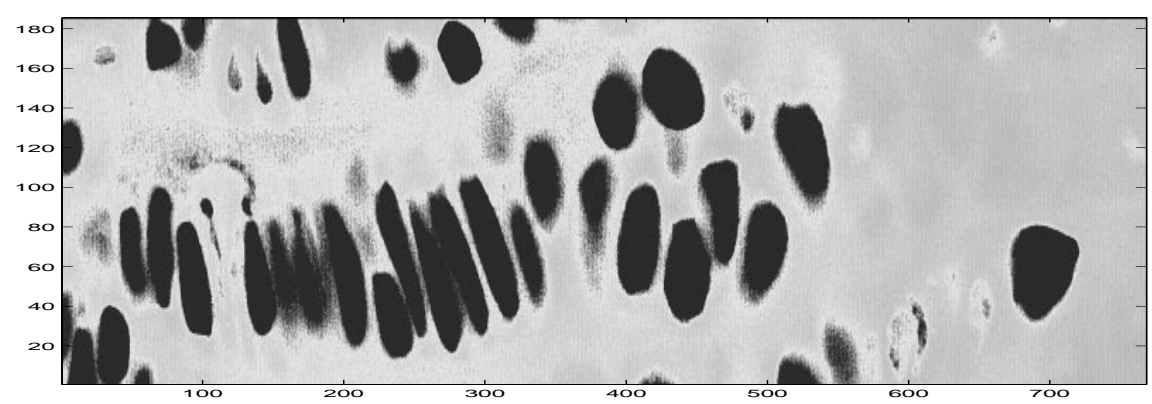

FIGURE 1: The image data.

reversible jump moves which alter the dimension of $\boldsymbol{x}$ by adding or removing a cell. Other very useful proposals are to split one cell into two or, conversely, to merge two cells into one. Such merge and split transitions are essential when there is uncertainty whether a feature in the data is one large cell or two smaller ones because moving between these two configurations by birth and death moves would require the chain to pass through intermediate states of very low probability. It is difficult to create rules for splitting or merging cells expressed in terms of the various shape, orientation and location parameters which are both efficient and inexpensive to compute. Crude proposals are generally available, but these tend to suffer high rejection rates since the current configuration has had ample opportunity to match the data well while an initial proposal of merged or split cells has not. Incorporating a fit to the data into the proposal itself is hard because of the computational cost of evaluating the posterior over a range of values for the proposal and of evaluating the reverse step to compute the acceptance probability. Rue and Hurn (1999) demonstrate one approach for such "data-matching" suitable for work with deformable polygonal templates, but the restriction to ellipses prevents us from using their approach here.

Figure 2 displays some possible split and merge type proposals for elliptical templates. These proposals are easily generated and use the common RJMCMC idea of averaging pairs of parameters when reducing dimension, and perturbing a parameter by a random amount in opposite directions to increase dimension. It is only sensible to attempt to merge cells which are close together and we control the distance between cells for which merging is considered by a parameter $\epsilon$. To illustrate how poorly the Markov chain can mix, we note results from runs of 20,000 steps of the sampler incorporating all move types: using a range of values for the separation parameter $\epsilon$, these runs produced between 1 and 7 accepted splits and no merges. 

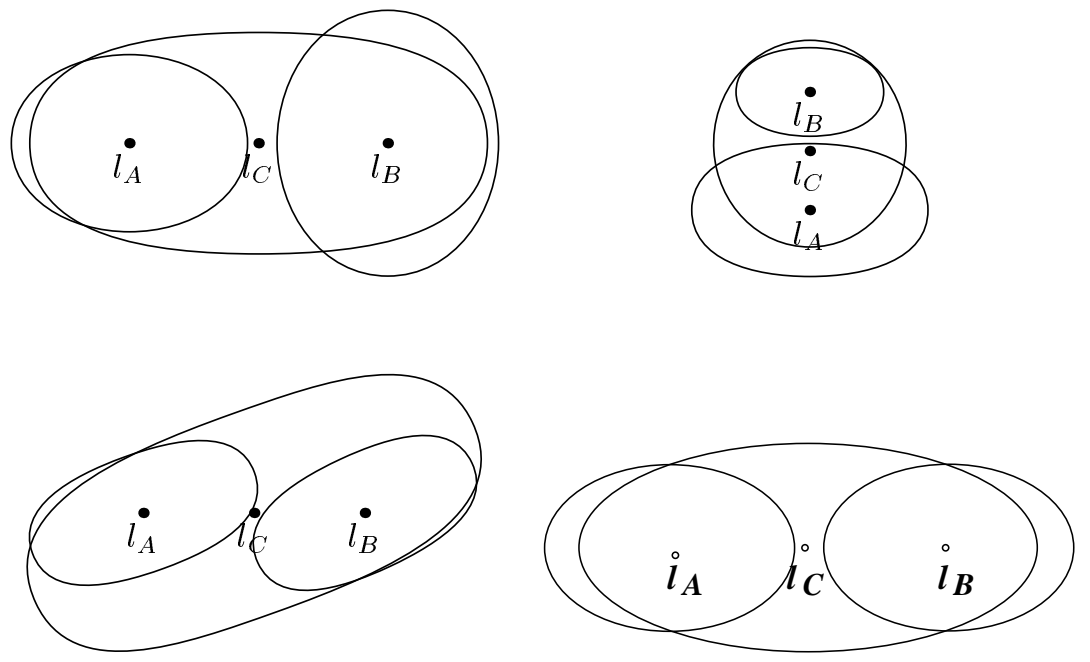

FIGURE 2: Some possible proposed split and merge moves

Our suggestion is to improve the data matching of a proposal before making the accept/reject decision. We apply tempering to the contribution to $\pi$ from the likelihood term to define the modified distribution

$$
\pi_{n}^{*}(\boldsymbol{x}) \propto \mathcal{L}^{\gamma}(\boldsymbol{y} \mid n, \boldsymbol{x}) p_{n}(\boldsymbol{x})
$$

Here $\gamma \in(0,1)$ and a good choice of $\gamma$ should give sufficient weight to the data to improve the data-fit of the final proposal $\boldsymbol{x}^{*}$ while limiting the effect of the low probability under $\pi$ of the initial proposal $\boldsymbol{x}^{\prime}$ on the final acceptance probability (4). In modifying $\boldsymbol{x}^{\prime}$ we use the usual fixed dimensional proposal types but with acceptance probabilities appropriate to the target distribution $\pi^{*}$. Note too that only the cells affected by the RJ proposal are changed, all others are held fixed.

In this example, $\pi$ and $\pi^{*}$ have the same support. In consequence, any proposal $\boldsymbol{x}^{\prime}$ with zero probability under $\pi$ (as a result of cell overlaps, for example) can be rejected straight away as we know $P\left(\boldsymbol{x}^{*}, \boldsymbol{x}^{\prime}\right)$ will be equal to zero in the hypothetical return route from a legal $\boldsymbol{x}^{*}$. It may sometimes be beneficial to extend the support of $\pi^{*}$, thereby allowing initial jumps to an illegal $\boldsymbol{x}^{\prime}$ to revert to legitimacy before reaching the final proposal $\boldsymbol{x}^{*}$. In order to encourage $\boldsymbol{x}^{\prime}$ to be in the state space of $\pi$, relatively little probability should be assigned to the extension of the state space. Similar ideas have been explored in a different context by Hurn, Rue and Sheehan (1999).

We have repeated experiments running 20,000 iterations of reversible jump 
Markov chains using various combinations of the tempering parameter $\gamma$ and the number of steps, $k$, from $\boldsymbol{x}^{\prime}$ to $\boldsymbol{x}^{*}$. Results, averaged over 3 replicates in each case, are listed in Table 1. The separation parameter $\epsilon$ was set equal to 500 and results should be compared with the 0 merges and 5 splits previously obtained for this case using unmodified proposals. Table 1 shows an increase in the number of successful splits and merges across parameter combinations. Similar improvements were also seen in runs for other values of the parameter $\epsilon$. There is no significance evidence of improvement when $k$ is increased from 50 to 300 , suggesting that adequate datamatching occurs quite quickly and a low value of $k$ is sufficient. There is welcome robustness to the choice of the tempering parameter $\gamma$ : values between 0.1 and 0.001 seem effective here. The success at very small values of $\gamma$ is perhaps surprising but can be attributed to the strong signal in this problem which leads to a highly modal target distribution $\pi$. Some pairs of image configurations during the RJMCMC runs are shown in Figure 3 to illustrate the scale of the changes. In each pair, the arrow indicates the area about to be affected.

The modified method has virtually no extra programming cost but it does require slightly greater computation due to the $k$ extra steps from $\boldsymbol{x}^{\prime}$ to $\boldsymbol{x}^{*}$ in modifying proposals. Since only split and merge proposals are affected, and the fixed dimensional moves within these types are not as costly as the moves between dimensions, the run time does not increase anywhere near a factor of $k$. Nevertheless, the modified method has succeeded in making merge moves and has improved a sampler which was really not mixing at all. Although the acceptance rates remain painfully low, we now have greater confidence that the sampler is capable of moving from one dimension to another through these essential routes. For this extremely hard sampling problem, this is significant progress.

In conclusion, the approach suggested in this article is one way in which low acceptance rates for RJMCMC samplers could be improved. It is particularly suitable for problems where it is hard to design very efficient proposals directly, but where the modality of the target distribution gives clues as to why many proposals are rejected. As another application, consider mixture modelling where observations are allocated to an unknown number of components; proposing to split a component 


\begin{tabular}{||l|c|c|c|c|c|c||}
\hline \hline & \multicolumn{2}{|c|}{$k=50$} & \multicolumn{2}{c|}{$k=100$} & \multicolumn{2}{c||}{$k=300$} \\
\hline$\gamma$ & merges & splits & merges & splits & merges & splits \\
\hline \hline 0.5 & 2 & 4 & 1 & 1 & 2 & 4 \\
\hline 0.1 & 6 & 14 & 5 & 9 & 5 & 7 \\
\hline 0.01 & 3 & 3 & 10 & 5 & 10 & 4 \\
\hline 0.001 & 2 & 3 & 8 & 6 & 10 & 4 \\
\hline 0.0001 & 1 & 2 & 4 & 5 & 4 & 7 \\
\hline 0.000001 & 2 & 2 & 0 & 2 & 3 & 6 \\
\hline \hline
\end{tabular}

TABLE 1: The average number of accepted merges and splits for different values of $\gamma$ and $k$, averaged over 3 runs.

may only be acceptable if the observations are reasonably well allocated to the two new components. Although some extra running costs are incurred in the modified proposals, there is little additional programming outlay, as existing code may be used.

\section{REFERENCES}

Al-Awadhi, F., (2001), Statistical Image Analysis and Confocal Microscopy, PhD. Thesis, Department of Mathematical Sciences, University of Bath, UK.

Baddeley, A. and van Lieshout, M.N.M., (1993), Stochastic Geometry Models in High-level Vision, in K.V.Mardia and G.K. Kanji, eds. Statistics and Images, (Carfax Publishing, Abingdon), pp 231-256.

Brooks, S., Giudici, P. and Roberts, G., (2001), Efficient Construction of Reversible Jump MCMC Proposal Distributions, Technical Report, University of Cambridge.

Cappe, O., Robert, C.P. and Ryden, T., (2001), Birth and Death Jump Processes for Inference in Hidden Markov Models, Technical Report, University Paris IX.

Gilks, W., Richardson, S. and Spiegelhalter, D., (1995), Practical Markov Chain Monte Carlo, (Chapman and Hall, London).

Green, P.J., (1995), Reversible Jump MCMC Computation and Bayesian Model Determination, Biometrika, 82, 711-732. 

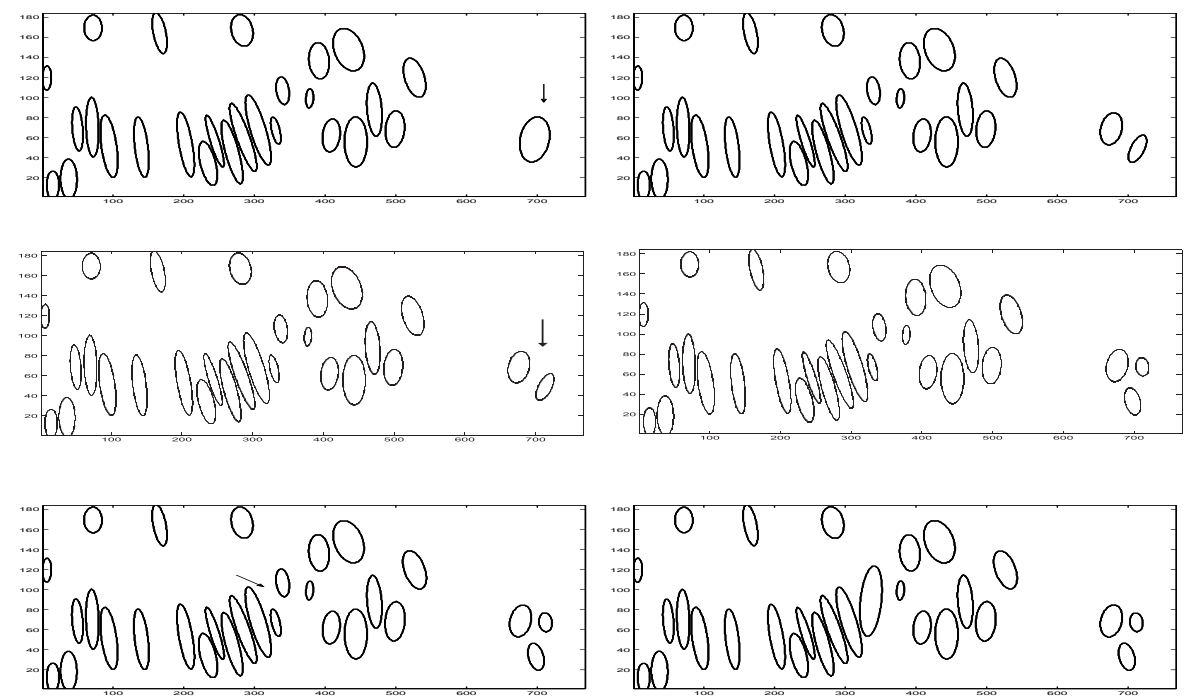

FIGURE 3: Several pairs of output configurations of the RJMCMC sampler using the modified proposal technique (the areas affected by successful split and merge moves are pointed out using arrows).

Green, P.J., (2002), Transdimensional Markov Chain Monte Carlo, in Highly Structured Stochastic Systems, to appear.

Green, P.J. and Mira, A., (2001), Delayed Rejection in Reversible Jump MetropolisHastings, Biometrika, 88, 1035-1053.

Hastings, W., (1970), Monte Carlo Sampling Methods using Markov Chains and their Applications, Biometrika, 57, 97-109.

Hurn, M.A., Rue, H. and Sheehan, N.A., (1999), Block Updating in Constrained Markov Chain Monte Carlo Sampling, Statistics and Probability Letters, 41, 353-361. Neal, R., (1996), Sampling from Multimodal Distributions using Tempered Transitions, Statistics and Computing, 6, 353-366.

Rue, H. and Hurn, M.A., (1999), Bayesian Object Identification, Biometrika, 86, 649660.

Stephens, M., (2000), Bayesian Analysis of Mixture Models with an Unknown Number of Components: An Alternative to Reversible Jump Methods, Annals of Statistics, 28, 40-74. 